\title{
A Numerical Analysis Research on Earlier Behavior of Molten Droplet Covered with Vapor Film at the Stage of Triggering and Propagation in Steam Explosion
}

\author{
Mingjun Zhong, Yankai Li, Meng Lin, Minghao Yuan, and Yanhua Yang \\ School of Nuclear Science and Engineering, Shanghai Jiaotong University, No. 800 Dongchuan Road, Shanghai 200240, China \\ Correspondence should be addressed to Meng Lin; linmeng@sjtu.edu.cn
}

Received 14 July 2014; Revised 7 September 2014; Accepted 9 September 2014; Published 12 October 2014

Academic Editor: Eugenijus Ušpuras

Copyright (c) 2014 Mingjun Zhong et al. This is an open access article distributed under the Creative Commons Attribution License, which permits unrestricted use, distribution, and reproduction in any medium, provided the original work is properly cited.

\begin{abstract}
When the molten fuel with high temperature falls into the cavity water, it will be dispersed into droplets which are covered with vapor films due to the rapid heat transfer with phase transition. This situation cannot be simply described by liquid-liquid or gasliquid systems. And there are no sufficient experimental studies on the behavior of droplet covered with vapor film because of the rapid reaction and the difficulty in capture of the film configuration. In this paper, a multiphase code with the volume of fluid (VOF) method is used to simulate the earlier behavior of droplet when vapor film exits. The earlier behavior is defined as behavior of the droplet before its disintegration. Thermal effect and pure hydrodynamic effect are, respectively, considered. The simulation results indicate that the film thickness and material density have significant effect on the earlier behavior of droplet. The situation assumed in Ciccarelli and Frost's model (1994) is observed in current simulation of earlier thermal droplet behavior. The effect of triggering pressure pulse on earlier hydrodynamic behavior is also discussed and it indicates that vapor film has little effect on the hydrodynamic droplet deformation when the intensity of the pressure pulse is very high.
\end{abstract}

\section{Introduction}

Steam explosion is recognized as one of the major concerns in nuclear power plant (NPP) safety analysis. Once vessel fails after melting of the reactor fuel and its relocation in the bottom of the reactor vessel, molten fuel could fall into a water pool. It will produce rapid heat transfer from the high temperature molten metal to the cold coolant with phase transition of the coolant, which may induce a vapor explosion and lead to an overpressurization.

The fuel and coolant interaction (FCI) process is commonly divided into four stages: coarse mixture, trigging, propagation, and expansion [1]. At the first stage, the continuous molten fuel with high temperature falls into the cavity water. Then the molten jet is dispersed into droplets. Due to rapid heat transfer and phase transition between the molten fuel and the liquid coolant, vapor films will be generated surrounding the droplets. At the triggering stage, effect of instability or the external pressure pulse could lead to collapse of the films. After that the coolant water directly contacts with the molten droplets, resulting in sudden heat transfer and evaporation of the coolant, which leads to the droplets' fragmentation. An increase of the melt surface area intensifies the heat and mass transfer and leads to local triggering regions with high pressure. At the propagation stage, the pressure spreads to surrounding areas. The droplets will break up into even smaller droplets or fragments. An increasing fuelcoolant area enhances the rates of heat and mass transfer and pressurization. At the expansion stage, pressure pulse with a high magnitude will result from the coupling of pressure wave propagation. It may potentially damage the reactor vessel structure and even the integrity of the containment. For FCI, many experimental studies based on a single droplet behavior have been done to investigate the mechanisms involving in droplet behavior [2-6]. According to previous works [2,3] the droplet fragmentation behavior is classically dominated by thermal effect and pure hydrodynamic effect.

For thermal behavior of the droplet, Kim and Corradini [7] postulated a model in which the water jet penetrates into drop and is retained under the melt surface. Subsequently 
evaporation of the penetrated water leads to the fragmentation of the drop. Ciccarelli and Frost [2] presented another mechanism where a local contact between water and droplet surface without penetration leads to a local pressurization that pushes part of the droplet, forming a filament. Koshizuka et al. [8] analyzed the thermal fragmentation by using the moving particle semi-implicit (MPS) method; normal boiling and rapid boiling based on the spontaneous nucleation theory were examined. The result showed that the spontaneous nucleation temperature played an important role in steam explosion. Zhou et al. [9] numerically simulated the thermal behavior of a single droplet, in which Kim's model was not observed unless the density of the molten droplet is hypothetically smaller than that of the coolant. In addition, Zhou et al. [9] also studied the effect of initial temperature of the melt and the coolant on thermal fragmentation.

The hydrodynamic behavior of the droplet is dominated by the drag and surface tension, which is described by Weber number (We $\left.=\rho U^{2} d / \sigma\right)$. Board et al. [10] proposed a theory of steam explosion postulating that the propagation step is due to hydrodynamic fragmentation. Experimental data of liquid droplet breakup in liquid-gas system were investigated by Pilch and Erdman [3] based on the initial Weber number. Five different breakup types were identified: (1) vibrational breakup regime; (2) bag breakup regime; (3) bag-and-stamen breakup regime; (4) sheet stripping regime where a thin sheet is continuously stripped from the surface of a deforming droplet; (5) and catastrophic breakup regime. Joseph et al. [11] take a photograph of viscous droplets breakup under the high speed airstream in a shock tube, and the phenomena of above modes 2 and 3 were observed at the very high Weber numbers. Hansson et al. [12] studied the microinteractions of a single droplet during the triggering phase of a steam explosion and found that the molten droplet experiences "preconditioning" before the fine fragmentation itself. Thakre et al. [13] simulated the molten droplet deformation using FLUENT, in which triggering pressure pulse is smaller than $5 \mathrm{MPa}$. The results of separate-effect study quantitatively demonstrated the importance of droplet velocity and melt properties on the melt droplet preconditioning. Zhong et al. [14] simulated the pure hydrodynamic droplet behavior without vapor film triggered by pressure pulse of almost $100 \mathrm{MPa}$. It was found that an increase of pressure pulse intensity will accelerate the completion of breakup process and the dimensionless breakup time is not strongly affected by bond number (Bo).

According to Berthoud [15], the trigger is the event that induces a transition between the coarse mixture stage and the propagation stage by locally initiating a rapid heat transfer and pressure rise. Once triggered, the pressure will propagate and escalate. During the escalation, the fragmentation mechanism changes from the thermal effect to a hydrodynamic effect due to the velocity difference between melt and coolant induced by high pressure propagation. It is suggested that, at the stage of triggering and the early stage of the propagation, thermal effect is the dominant factor in the droplet behavior because the intensity of the pressure pulse is weak; at the later stage of the propagation, the hydrodynamic effect will act as a dominant role because the amplitude of the pressure pulse is coupled with a large magnitude. Experimental study $[3,11]$ on the droplet hydrodynamic behavior was always carried out under nonboiling condition. Therefore the effect of vapor film on droplet hydrodynamic behavior was not considered, while experimental observation of the droplet thermal behavior cannot capture the film configuration precisely. In order to investigate the FCI fragmentation mechanism in more detail, a multicomposition homogeneous-phase code is used to simulate the earlier behaviors of droplet covered with vapor film. Thermal effect and pure hydrodynamic effect are considered separately, and effects of the film thickness, material density, and external pressure pulse are examined for parameter analyses.

\section{Physical Model and Numerical Methods}

2.1. Physical Model. Code used in the study is a homogeneous-phase (multicomposition), two-dimensional, and transient code, and the basic field equations are shown as follows.

Mass equation is

$$
\frac{\partial \rho}{\partial t}+\frac{\partial \rho u}{\partial x}+\frac{\partial \rho v}{\partial y}=0
$$

Momentum equation is

$$
\begin{aligned}
& \frac{\partial \rho u}{\partial t}+\frac{\partial \rho u u}{\partial x}+\frac{\partial \rho u v}{\partial y} \\
& =\frac{\partial}{\partial x}\left(\mu \frac{\partial u}{\partial x}\right)+\frac{\partial}{\partial y}\left(\mu \frac{\partial u}{\partial y}\right)-\frac{\partial p}{\partial x}+\rho g_{x}+\sigma \kappa \delta_{s} \vec{n}_{x} \\
& \frac{\partial \rho v}{\partial t}+\frac{\partial \rho v u}{\partial x}+\frac{\partial \rho v v}{\partial y} \\
& =\frac{\partial}{\partial x}\left(\mu \frac{\partial v}{\partial x}\right)+\frac{\partial}{\partial y}\left(\mu \frac{\partial v}{\partial y}\right)-\frac{\partial p}{\partial y}+\rho g_{y}+\sigma \kappa \delta_{s} \vec{n}_{y} .
\end{aligned}
$$

Energy equation is

$$
\begin{gathered}
\frac{\partial \rho C_{P} T}{\partial t}+\frac{\partial \rho C_{P} T u}{\partial x}+\frac{\partial \rho C_{P} T v}{\partial y} \\
=\frac{\partial}{\partial x}\left(\lambda \frac{\partial T}{\partial x}\right)+\frac{\partial}{\partial y}\left(\lambda \frac{\partial T}{\partial y}\right)+\phi .
\end{gathered}
$$

Melt, coolant, and vapor are included and simulated in current study. The properties in the cell are determined by volume fraction $C_{i}$ of each phase as below:

$$
\begin{aligned}
\rho & =\sum C_{i} \rho_{i}, \\
\mu & =\sum C_{i} \mu_{i}, \\
\lambda & =\sum C_{i} \lambda_{i}, \\
C_{P} & =\sum C_{i} C_{P i} .
\end{aligned}
$$

The term of the surface tension $\left(\sigma \kappa \delta_{s} \vec{n}\right)$ in the momentum equation is treated with continuum surface force (CSF) model 
[16] which has been used by various researchers $[17,18] . \phi$ in energy equation is a source for the latent heat due to phase change at the interface. A simplifying assumption is introduced in current simulation. It is assumed that evaporation or condensation occurs at the vapor film interface and the interface temperature is equal to the saturation temperature corresponding to the system pressure. Therefore energy transfer at the film interface during phase change with radiation heat transfer can be written as

$$
m h_{l v}=\vec{q}_{l}-\overrightarrow{q_{v}}+q_{r}=\left(\left.\lambda_{l} \frac{\partial T}{\partial \vec{n}}\right|_{l}-\left.\lambda_{v} \frac{\partial T}{\partial \vec{n}}\right|_{v}\right)+q_{r}
$$

Here $\vec{n}$ is the unit normal vector to the interface. $\overrightarrow{q_{l}}$ and $\overrightarrow{q_{v}}$ are heat flux, respectively, in liquid side and vapor side of the interface. $q_{r}$ is the radiation energy participating in phase change. Because the vapor film is always very thin, the vapor is regarded as nonparticipating media for radiation and the radiant energy is assumed to be absorbed at the interface and acts as an energy source for evaporation. The radiant heat flux is expressed as

$$
q_{r}=\varepsilon \sigma_{b} T_{d}^{4}
$$

2.2. Numerical Methods. Finite volume method is used to discretize the governing equations and pressure-velocity linkage is resolved with the SIMPLE algorithm. The current numerical algorithm and VOF method were used in the simulation of Yuan et al. [19] and Zhong et al. [14] and performed well.

2.2.1. Phase Transport and VOF Method. In order to obtain the distribution of different composition volume fraction $C_{i}$, a transport equation as below is solved by VOF method:

$$
\frac{\partial C_{i}}{\partial t}+\frac{\partial C_{i} u}{\partial x}+\frac{\partial C_{i} v}{\partial y}=\Gamma_{i}
$$

$\Gamma_{i}$ is a source term for phase change of liquid coolant, which can be obtained by (5). Through calculation of the fluxes of liquid volume through all cell faces within a time step, a new value of $C_{i}$ can be obtained. The VOF method is used to reconstruct interface and obtain the free-surface geometry information. There are several different VOF algorithms with different reconstruction schemes, such as SOLAVOF [20], FLAIR method [21], and Youngs VOF [22]. Among those methods, Youngs VOF method is proved to perform well in most situations and is employed in our simulations. Youngs VOF uses a line segment (in two dimensions) defined by a slope and intercept to represent the interface in a mixture cell, which is usually called PLIC (Piecewise Linear Interface Construction).

2.2.2. Method for Phase Change at Interface. When phase change of coolant occurs at the interface, there will be some difficulties in the calculation of the flux of fluid volume through a cell face with VOF/PLIC algorithm. As shown in Figure 1, the mixture cell contains both vapor and liquid, and evaporation exists at the interface at the same time. It is assumed that all the velocities are zero except the one

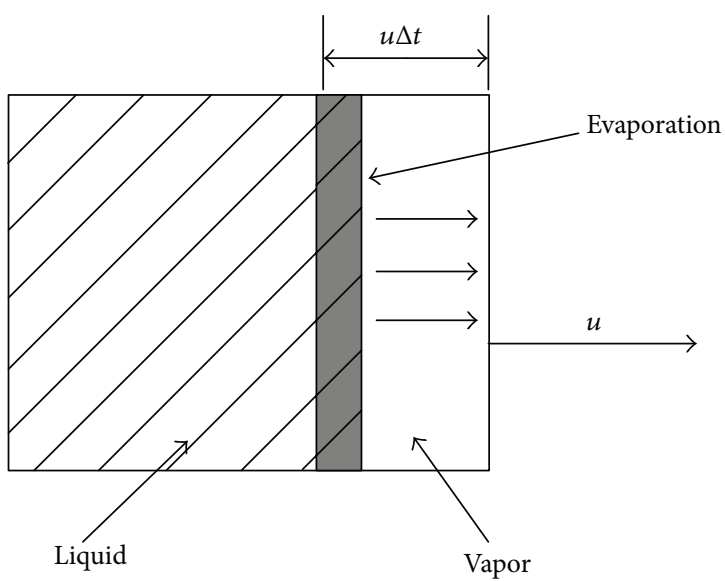

FIGURE 1: A typical mixture cell and the configuration of interfacial flux.

located at the right face of the cell. Because vapor is generated continuously at the interface, the contravariant velocity at the right face is toward outside of the cell. With the VOF/PLIC method, which tracks the volume fraction of liquid, for instance, the flux of volume of liquid through the right face of the cell is calculated by the shaded area, as shown in Figure 1. It is obvious that this flux is not really transported, because in such situation, no liquid is transported through the right face of the cell, and all the fluid passing the right face is vapor. Faced with this kind of problem a simplified treatment has been taken [19]. As shown in Figure 1, vapor is generated continuously at the free interface. The volume expansion or contraction due to mass exchange is all moved from the mixture cell where phase change really occurs to the nearest vapor cell. With this strategy, the velocity field is divergence free for all the mixture cells. Thus, the VOF/PLIC method is applicable to the mixture cells without any modifications to the algorithm except that the volume of liquid which has evaporated should be subtracted from the mixture cell.

\section{Simulation and Discussion}

A two-dimensional domain is used due to less computational effort and axial symmetric assumption. A rectangular domain of $80 \mathrm{~mm}$ (horizontal) $* 40 \mathrm{~mm}$ (vertical) is applied with a structured orthogonal grid in a cylindrical coordinate system. After the grid sensitivity study, 201 and 601 cells are, respectively, arranged in radial and axial directions finally. As illustrated in Figure 2, the red region represents the initial location of the molten droplet. The minimum grid length scale around the molten droplet is $1.5 \times 10^{-5} \mathrm{~m}$, and the grid of the melt-vapor-coolant interface is refined enough to capture the interfacial behavior.

3.1. Earlier Thermal Behavior of a Droplet. Ciccarelli and Frost [2] suggested that the unstable vapor film around a droplet would collapse by a trigger of external pressure pulse. After the direct contact of droplet and coolant, fine fragmentation could happen. In present study, the thermal behavior 


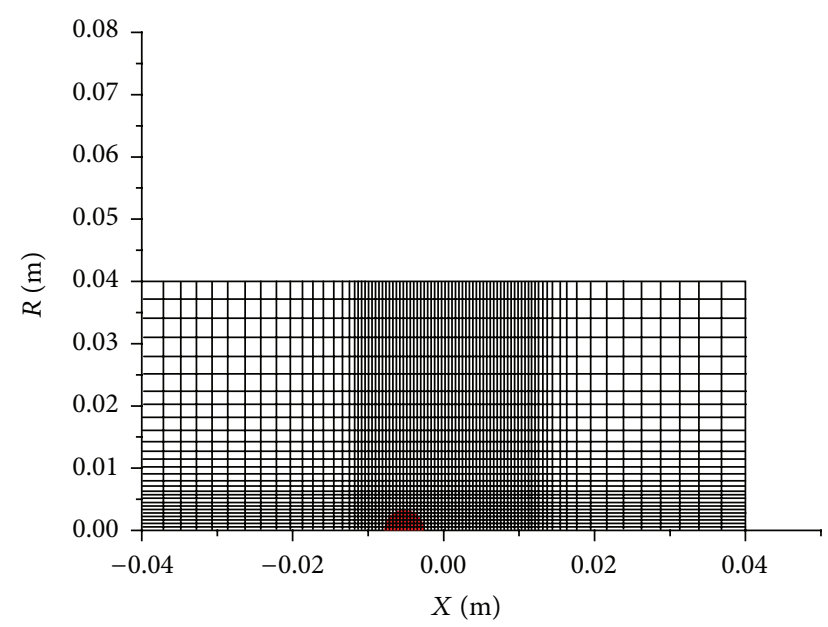

FIgURE 2: Configuration and grids of the calculation domain.

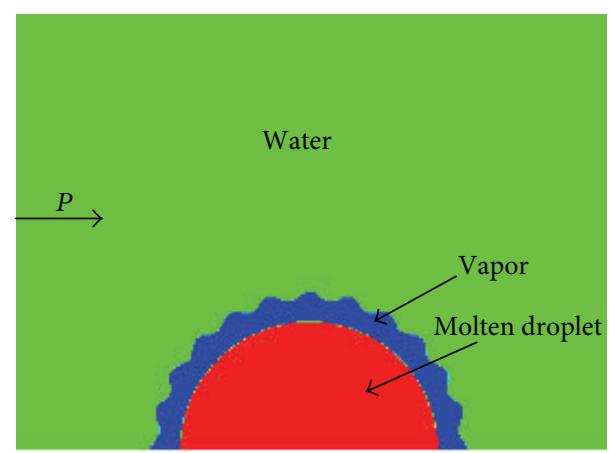

FIGURE 3: Configuration of the thermal droplet behavior calculation.

of a droplet is focused on the vapor film collapse, the direct contact of droplet and coolant, and the molten droplet interface behavior, which can be regarded as earlier behaviors in thermal fragmentation. Referring to the investigation of forced film boiling of a molten droplet in steam explosion [23], the film thickness is generally less than $0.5 \mathrm{~mm}$ at the stagnant point, which is used as a reference for film thickness in the current simulation.

At the stage of triggering, relative flow between each component is not strong and the droplets will be probably triggered by the pressure propagation generated from a droplet which has been triggered. Additionally in some small scale experiments [24,25], velocity of the ambient liquid was small and an external pressure pulse was used to trigger the process. Therefore in this calculation, a stationary molten droplet with a diameter of $3 \mathrm{~mm}$ is uniformly covered by an unstable vapor film shown in Figure 3. A trigger of pressure pulse shown in Figure 4 is provided at the left boundary of the calculation domain. The peak of the pulse $\left(P_{\max }\right)$ is assumed to be $4 \mathrm{MPa}$, which will induce a relatively low ambient liquid velocity corresponding to the experiment [2]. The initial temperature of the molten droplet is $1073 \mathrm{~K}$ and coolant water is set to a hypothetical saturation temperature of $373 \mathrm{~K}$. The initial droplet diameter and initial temperature of the material are

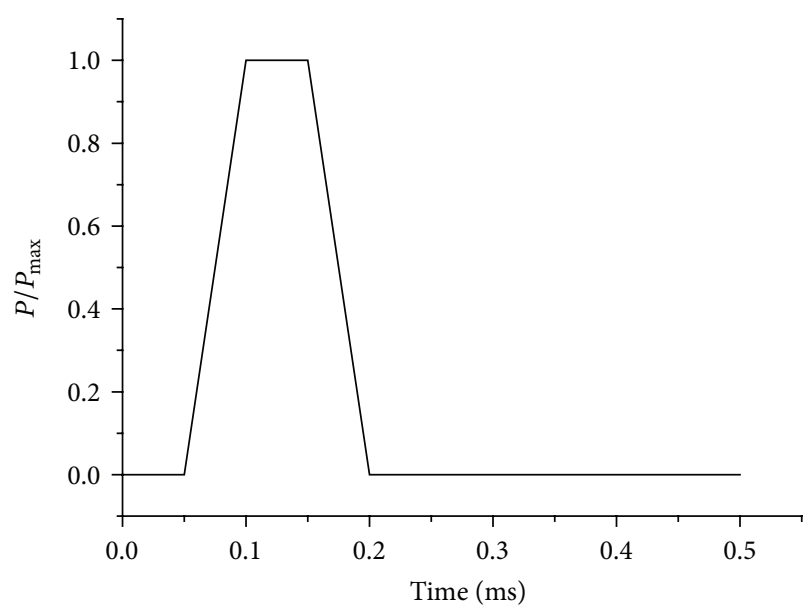

FIGURE 4: Pressure pulse profile in the calculation.

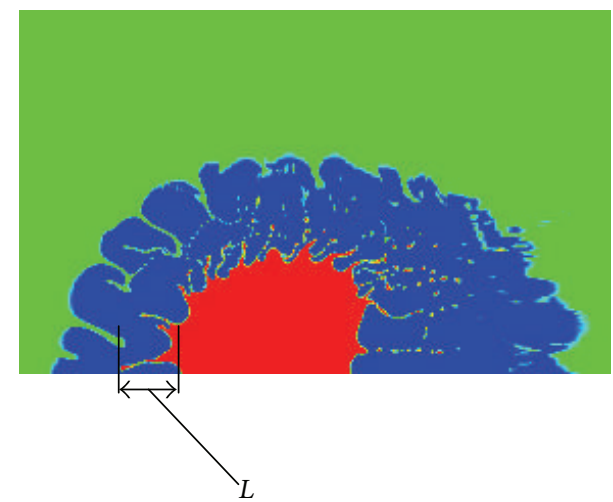

FIGURE 5: The parameter introduced for thermal droplet behavior analysis.

similar to the experiment by Ciccarelli and Frost [2], in which the material of droplet is molten tin. Ciccarelli and Frost [2] indicate that small ripples $(0.5 \mathrm{~mm})$ were presented on the vapor film before film collapse. Therefore the thickness of vapor film is initially assumed as

$$
\delta_{\text {film }}=\delta_{\text {film } 0}+A \cos \left(\frac{2 \pi x}{\lambda}\right) .
$$

Here, $A$ is amplitude of the perturbation wave of the vapor film: $A=0.5 \mathrm{~mm} ; \lambda$ is the hypothetical wave length: $\lambda=0.47 \mathrm{~mm} . \delta_{\text {film } 0}$ is the initial vapor film thickness without perturbation. For thermal behavior at earlier stage, the formation of filaments is an important phenomenon. Therefore, the length of the first filament generated on the droplet as shown in Figure 5 is introduced to evaluate the process. In current study, first filament on the left side of the droplet is representative, because the position where it generates is predominated by normal flow field and affected by strong Taylor instability. According to the results of simulations, the first filament grows faster than other ones and is easy to measure. 


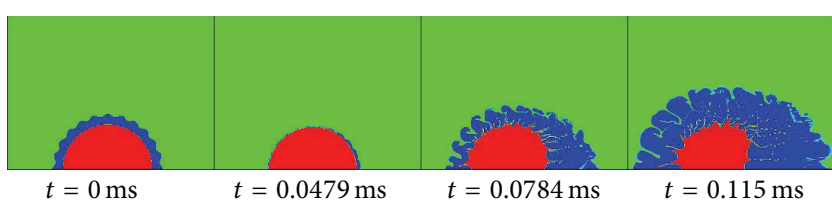

Figure 6: Evolution of a droplet with an unstable vapor film $\left(\rho_{d}=\right.$ $\left.7000 \mathrm{~kg} / \mathrm{m}^{3}\right)$.

3.1.1. Effect of Vapor Film Thickness on the Thermal Behavior. In this calculation, a three-phase system (melt-vapor-coolant) considering energy transfer is carried out. The density of the molten droplet is $7000 \mathrm{~kg} / \mathrm{m}^{3}$ (material: tin simulant), the surface tension is $0.52 \mathrm{~N} / \mathrm{m}$, and the conductivity is $60 \mathrm{~W} / \mathrm{m} \cdot \mathrm{K}$. An unstable vapor film with $\delta_{\text {film } 0}=0.3 \mathrm{~mm}$ is initialized surrounding the droplet. The evolution of the thermal droplet behavior is shown in Figure 6. As a result of man-made outside pressure wave, the vapor film collapses at $0.0479 \mathrm{~ms}$ bringing a direct contact of water and the droplet. The initial droplet-coolant direct contact after vapor film collapse induces rapid heat transfer leading to the bubble expansion. At the contact locations, local generation of high pressure causes the formation of surface waves and pushes the filaments from the droplet surface to outside. During this simulation process, the penetration of water jet assumed Kim's model [7] is not observed. The concept of Ciccarelli and Frost [2] and their experimental observation are shown in Figure 7. The basic features of filament generation simulated agree with the experimental observation.

In order to analyze the effect of vapor film thickness, two additional cases with $\delta_{\text {film } 0}=0.5 \mathrm{~mm}$ and $\delta_{\text {film } 0}=0.7 \mathrm{~mm}$ are also examined. A comparison of the aspect ratio of $L / R_{0}$ with respect to time is presented in Figure 8. It indicates that growth rate of the filament is higher for the molten droplet with thicker film. It could be because that a larger amount of vapor is compressed when the film collapses, a higher pressure is generated at the spot of contact, and then more filament is pushed out from the droplet surface.

3.1.2. Effect of Density Ratio on the Thermal Behavior. In order to evaluate the effect of density ratio on the thermal behavior of a droplet, three molten droplet materials with different densities of $10000 \mathrm{~kg} / \mathrm{m}^{3}$ (material: UO2 simulant), $7000 \mathrm{~kg} / \mathrm{m}^{3}$ (material: tin simulant), and $3000 \mathrm{~kg} / \mathrm{m}^{3}$ (material: aluminium simulant) are introduced. The corresponding density ratios of coolant water to droplet are $1 / 10,1 / 7$, and $1 / 3$, respectively. Other properties are the same: the surface tension is $0.52 \mathrm{~N} / \mathrm{m}$ and the conductivity is $60 \mathrm{~W} / \mathrm{m} \cdot \mathrm{K}$. The surface tension is kept constant in each case and the triggering pressure pulse is the same. The thermal behavior of the droplet with density of $3000 \mathrm{~kg} / \mathrm{m}^{3}$ is presented in Figure 9 and still no obvious penetration of water is observed. A further comparison of filament growth rate in terms of aspect ratio of $L / R_{0}$ with respect to time is shown in Figure 10. It is clear that the filament growth rate is higher for the denser droplet.
In Zhou's [9] simulation (Figure 11(b)), when the density of the molten droplet is smaller than that of coolant, a similar behavior of water penetration consistent with Kim's model [7] (Figure 11(a)) will occur. But this hypothetical density ratio is not realistic in real reactor severe accident situation. Therefore it is believed that the model of Ciccarelli and Frost [2] is a more reasonable one to describe the thermal behavior of a molten droplet.

3.2. Earlier Hydrodynamic Behavior of a Droplet. For hydrodynamic droplet behavior analysis, the study is mainly focused on the deformation of the droplet and stripping of the vapor film, which can be considered as earlier behaviors in hydrodynamic fragmentation. In order to simplify and abstract the progress, the heat transfer and the phase change are not considered in the hydrodynamic deformation simulation. Considering the characteristic of short time scale in film boiling evaporation, it is believed that the isothermal simplification is acceptable.

In order to validate the current methodology of hydrodynamic study, the experimental data of Boiko and Poplavski [26] is used for comparison. Referring to the experiment, the material of the droplet is water and the ambient fluid is gas. The same Weber number (We $=400$ ) is applied in the simulation. Figure 12 presents the results of the simulation and the experiment. The basic feature of the droplet deformation and stripping phenomenon obtained in the calculation agrees with the experimental observation. Figure 13 shows the comparison of the simulation results with the experimental data in terms of the aspect ratio of $d_{\max } / d_{0}$ with respect to time. The aspect ratio of $d_{\max } / d_{0}$ is defined as the max diameter of the deforming droplet divided by the initial diameter. In this calculation, $d_{\max } / d_{0}$ will be selected as a parameter to compare with the experimental data. Figure 13 illustrates that the trend predicted by the simulation result agrees with the experimental data in an acceptable range.

For further comparison, the experimental data of Ciccarelli and Frost [2] who investigated the fragmentation processes of single drops of molten metal immersed in water is applied. The simulation is carried out at the condition similar to the experiment $(\mathrm{We}=8500)$. In the simulation the density of the molten droplet is $7000 \mathrm{~kg} / \mathrm{m}^{3}$; the surface tension is $0.52 \mathrm{~N} / \mathrm{m}$. Figure 14 shows the comparison of the simulation result with the experimental data, which indicates that the prediction of the simulation agrees with the experiment well.

\subsubsection{Effect of Vapor Film Thickness on the Hydrodynamic} Behavior. A three-phase system (melt-vapor-coolant) is used for evaluation of hydrodynamic behavior of a droplet covered with uniform vapor film. In the calculation, the density of the molten droplet is $10000 \mathrm{~kg} / \mathrm{m}^{3}$ and its surface tension is $0.52 \mathrm{~N} / \mathrm{m}$. A vapor film with the thickness of $0.5 \mathrm{~mm}$ is initialized surrounding the droplet with the diameter of $5 \mathrm{~mm}$. The ambient coolant is water and an initial velocity $2.5 \mathrm{~m} / \mathrm{s}$ is given leftward to both the vapor film and the droplet. Figure 15 shows the evolution of the droplet surrounded by 


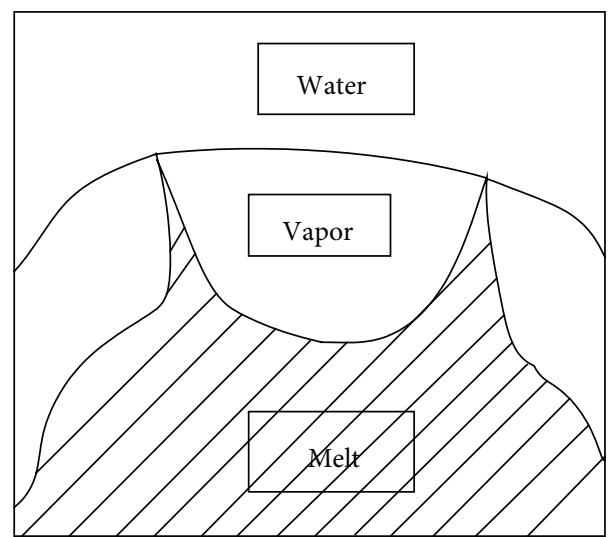

(a)

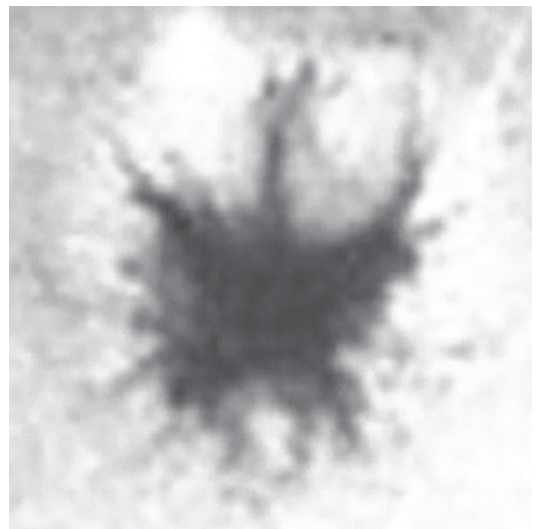

(b)

FIgURE 7: Description of Ciccarelli and Frost's model [2] (a) and the experimental observation (b).

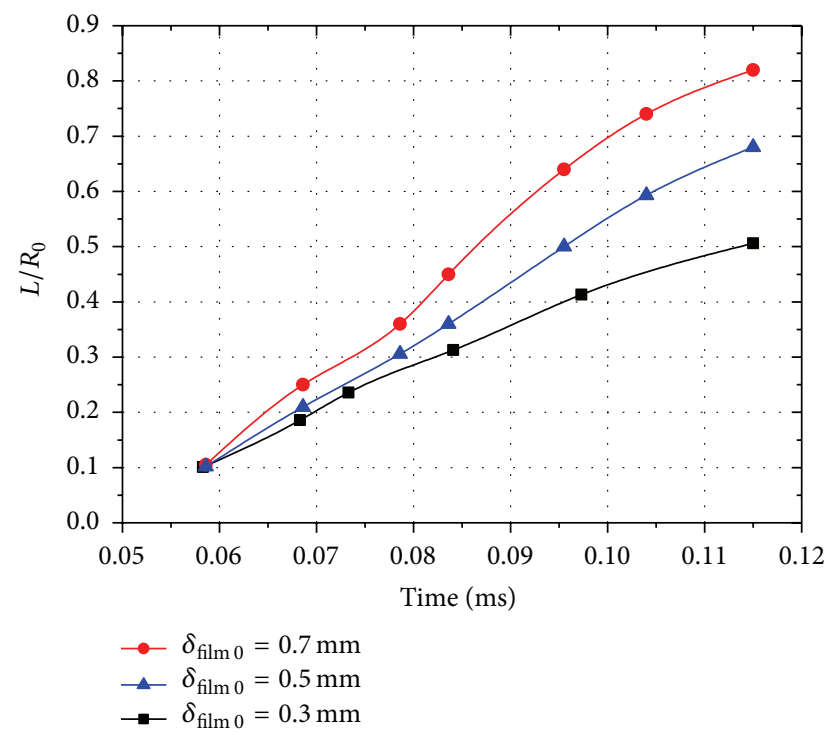

FIGURE 8: Aspect ratio of filament length to droplet radius with time for different film thicknesses.

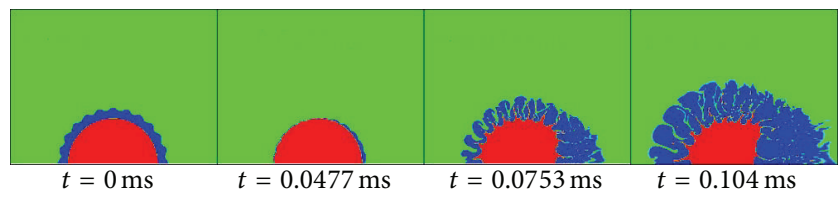

FIGURE 9: Evolution of a droplet with an unstable vapor film $\left(\rho_{d}=\right.$ $\left.3000 \mathrm{~kg} / \mathrm{m}^{3}\right)$.

a vapor film. As a result, the process can be divided into two stages. At the first stage, the droplet tries to get through the vapor film but the whole surface of the droplet is still covered by the vapor. During this stage, the droplet deforms slightly. After $1.6 \mathrm{~ms}$ the front of the droplet penetrates the film and at $3.2 \mathrm{~ms}$ the main part of the droplet has been exposed to the ambient coolant. At the second stage, the droplet has

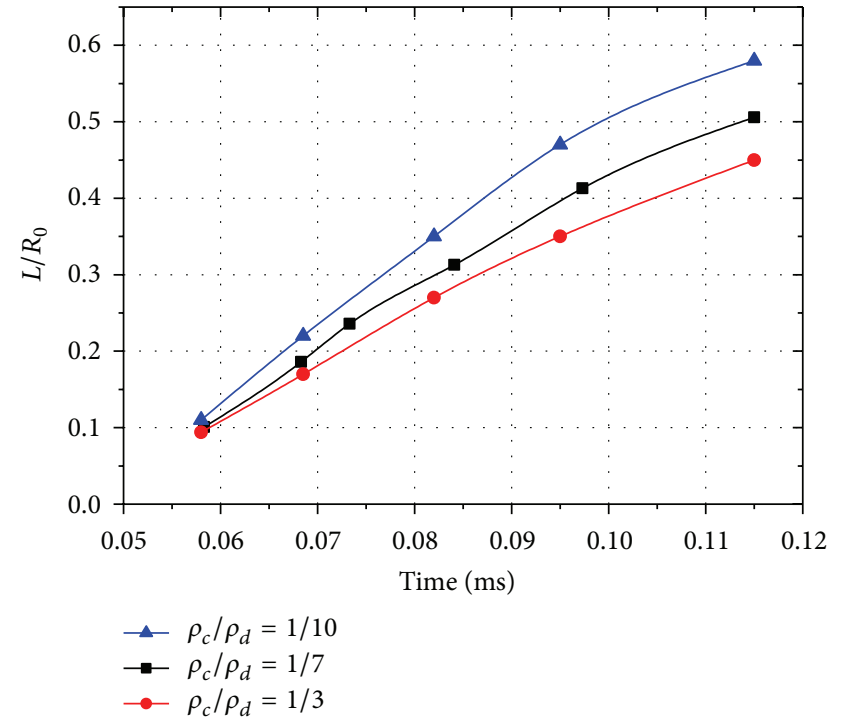

FIGURE 10: Aspect ratio of filament length to droplet radius with time for different droplet densities.

penetrated the vapor film and deforms faster. The vapor accumulates in the cavity of C-shaped droplet.

Behaviors of droplets with different thicknesses of vapor films have been examined. Figure 16 shows the comparison of deformation rate in terms of aspect ratio of $A / A_{0}$ with respect to time. The aspect ratio of $A / A_{0}$ is defined as the surface area of the droplet to the initial value, which is more accurate to describe deformation of the droplet. It can be seen that the deformation rates are close at the beginning and then droplets with thinner films deform faster. Referring to the comparison of the cases of droplets with and without vapor film $\left(\delta_{\text {film }}=\right.$ $0.0 \mathrm{~mm}$ ), it is believed that the presence of a vapor film will suppress the droplet deformation. It is because of the fact that the vapor film decreases the relative velocity of the droplet and the ambient liquid when the droplet penetrates the film. 


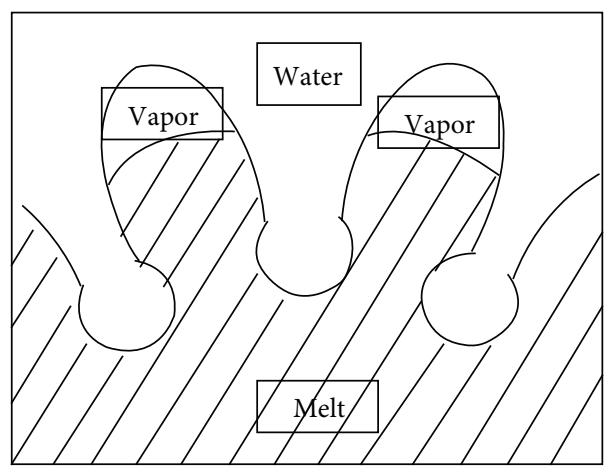

(a)

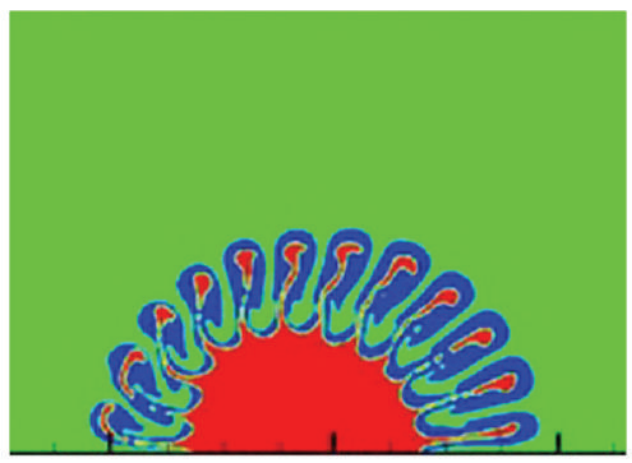

(b)

FIGURE 11: Description of Kim's model [7] (a) and Zhou's simulation result [9] (b).

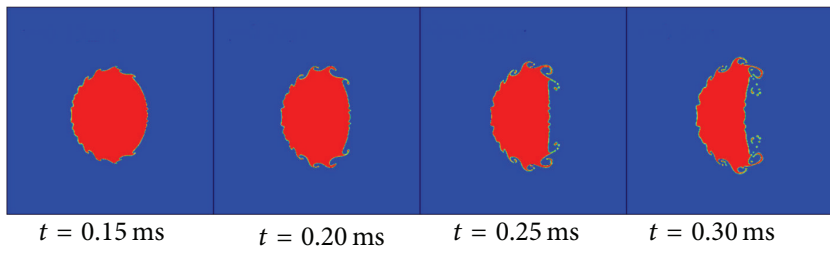

(a)

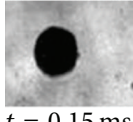

$t=0.15 \mathrm{~ms}$

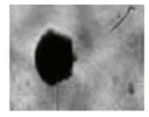

$t=0.21 \mathrm{~ms}$

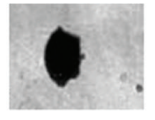

$t=0.24 \mathrm{~ms}$

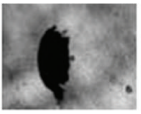

$t=0.30 \mathrm{~ms}$

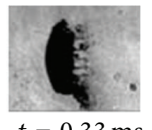

$t=0.33 \mathrm{~ms}$ (b)

FIGURE 12: Evolution of a simulated droplet (a) and experimental observation of Boiko (b).

3.2.2. Effect of Density Ratio on the Hydrodynamic Behavior. In order to evaluate the effect of density ratio on the behavior of the droplet, two different hypothetical droplet materials with densities of $10000 \mathrm{~kg} / \mathrm{m}^{3}$ and $7000 \mathrm{~kg} / \mathrm{m}^{3}$ are introduced. The corresponding density ratios of coolant water to droplets are $1 / 10$ and $1 / 7$, respectively. The surface tension is kept constant in each case and the initial velocity imposed on the droplet and the film is the same. The comparison of deformation rate in terms of aspect ratio of $A / A_{0}$ with respect to time is shown in Figure 17. It is found that the deformation rates are nearly equal before $2 \mathrm{~ms}$ and then the denser droplet deforms faster. It can be concluded that the denser droplets experience larger deformation.

3.2.3. Effect of Trigging Pressure. For a more realistic case of pressure propagation during the steam explosion, an external trigging pressure pulse is provided in the current simulation. The pressure pulse shown in Figure 4 is set at the left boundary of the calculation domain. The density of the molten droplet is $7000 \mathrm{~kg} / \mathrm{m}^{3}$; the surface tension is $0.52 \mathrm{~N} / \mathrm{m}$. Figure 18 gives the comparison of deformation rates of the droplets with and without vapor films in different pressure pulse peaks $(10 \mathrm{MPa}$ and $5 \mathrm{MPa})$. It demonstrates that an increase of

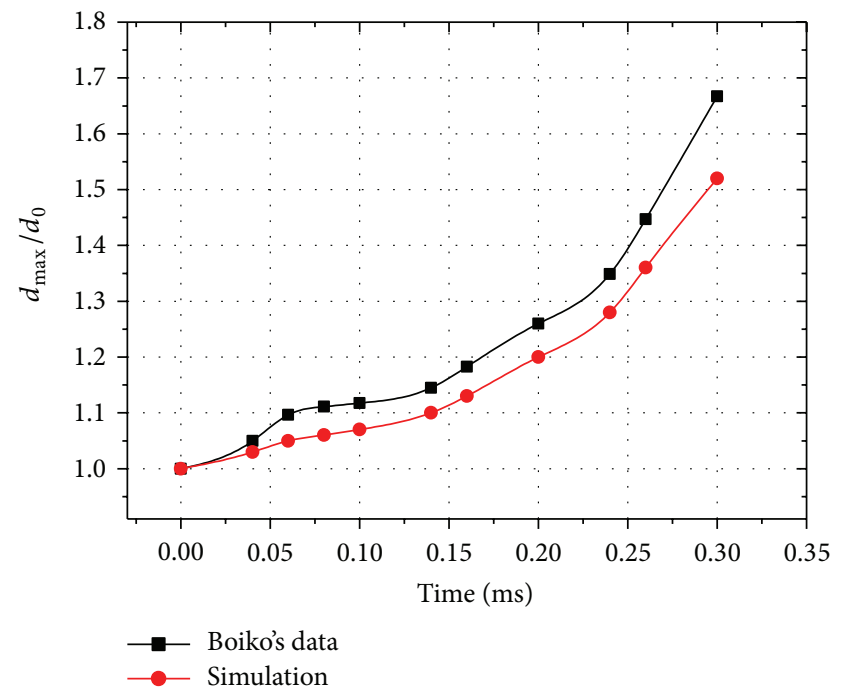

FIGURE 13: Comparison of numerical results with experimental data $[20]$.

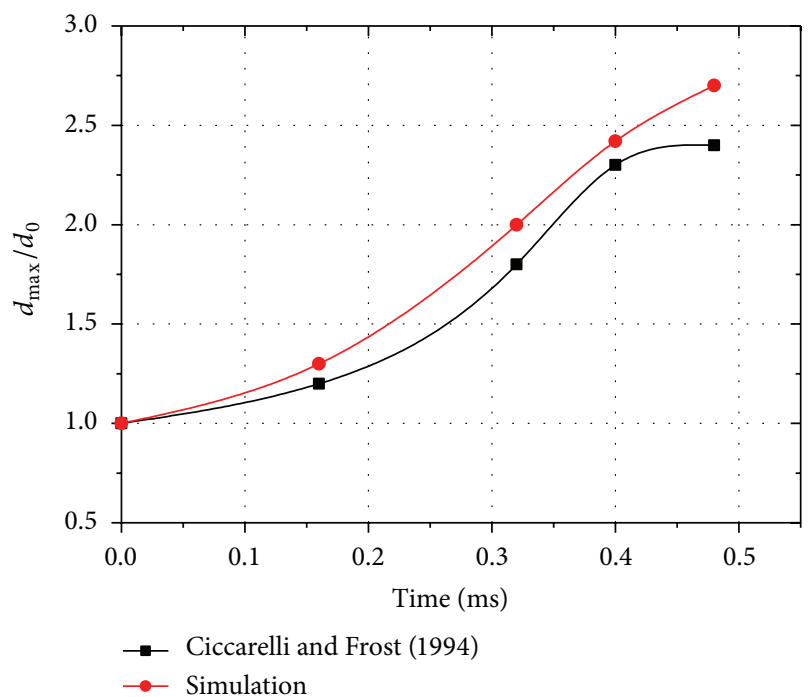

FIGURE 14: Comparison of numerical results with experimental data [2]. 


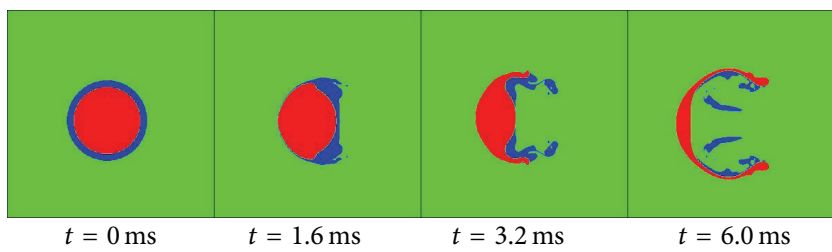

FIGURE 15: Evolution of a droplet with a vapor film (film thickness: $0.5 \mathrm{~mm})$.

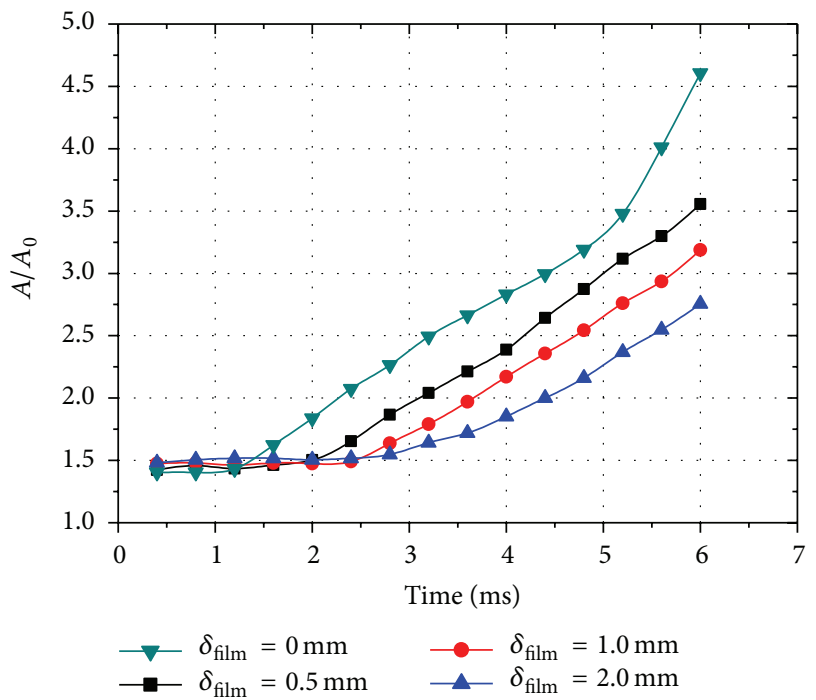

FIGURE 16: Aspect ratio of droplet surface area with time for different film thicknesses.

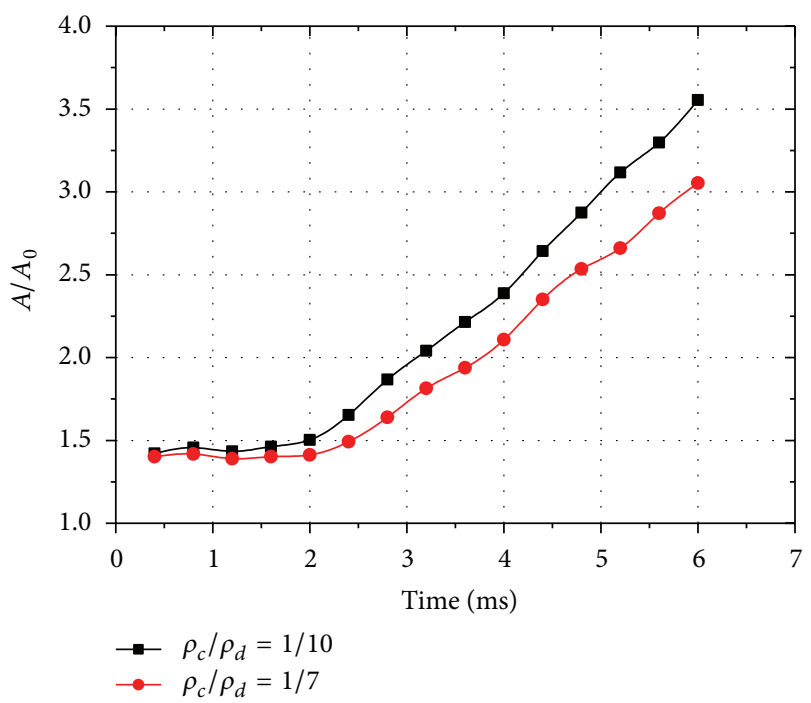

FIGURE 17: Aspect ratio of droplet diameter with time for different droplet densities.

pressure pulse will accelerate the droplet evolution and the vapor film will suppress the process. As the pressure pulse amplitude increases, the effect of vapor film becomes weak. In real steam explosion situation and considering the later stage of propagation, the pressure pulse will be coupled to

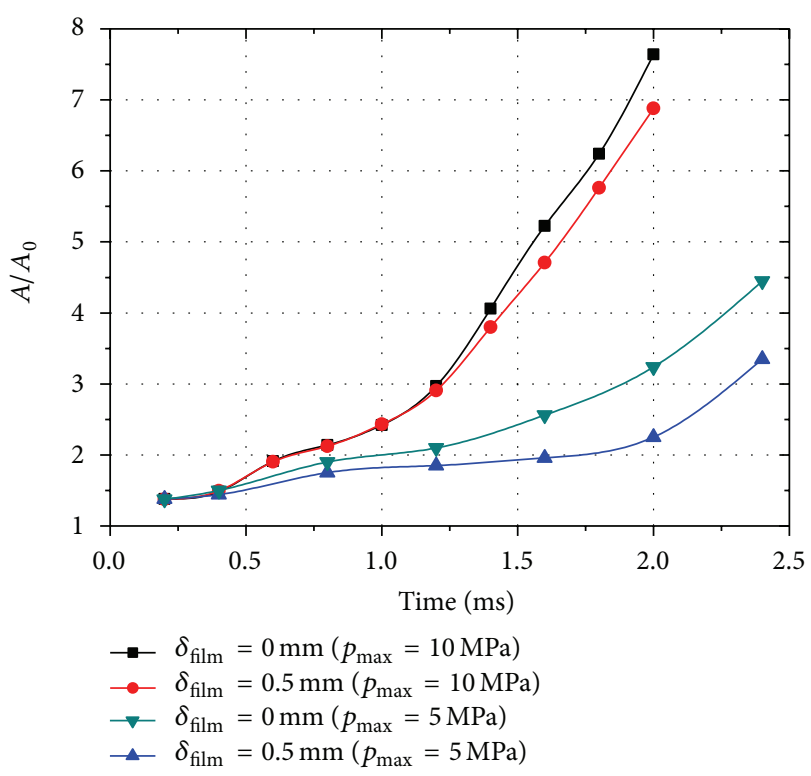

FIGURE 18: Aspect ratio of droplet diameter with time for the droplets with and without film (the peaks of pressure pulses are $10 \mathrm{MPa}$ and $5 \mathrm{MPa}$ ).

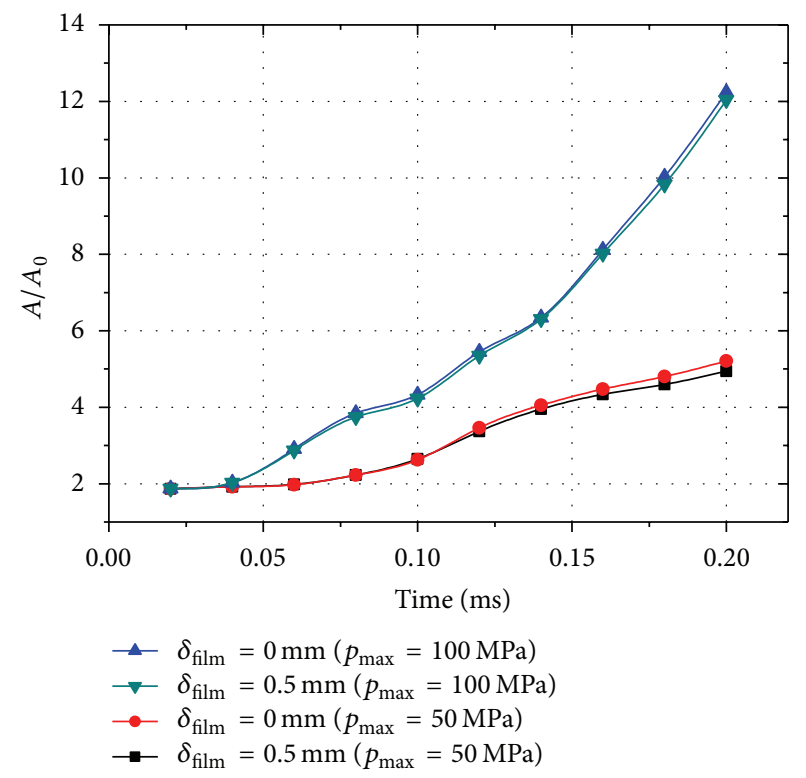

FIGURE 19: Aspect ratio of droplet diameter with time for the droplets with and without film (the peaks of pressure pulses are $100 \mathrm{MPa}$ and $50 \mathrm{MPa}$ ).

a high intensity which may exceed $100 \mathrm{MPa}$ [27]; therefore a pressure pulse with peak value of $100 \mathrm{MPa}$ and a pressure pulse with peak value of $50 \mathrm{MPa}$ are introduced. Figure 19 gives the comparison of deformation rates of the droplets with and without vapor films. It indicates that under a high pressure pulse the vapor film has little effect on the droplet deformation. It is believed that the vapor film could be neglected during the later stage of pressure propagation in a real NPP steam explosion situation. 


\section{Conclusions}

In order to investigate the FCI fragmentation mechanism in more detail, a multicomposition homogeneous-phase code with VOF method is used to simulate the earlier behavior of molten droplet covered with vapor film. Experimental data and observation $[2,20]$ are used to validate the current code. Thermal effect and pure hydrodynamic effect are considered, respectively, in present study. For parameter analyses, effects of the vapor film thickness and the material density are analyzed, and the effect of triggering pressure pulse on hydrodynamic behavior is also discussed.

In FCI, at the stage of triggering and the early stage of the propagation, thermal effect is the dominant factor in the droplet. For the earlier thermal behavior, the simulation results show that the growth rate of the filament is higher for the molten droplet with a thicker film once the film collapsed. At the same time, the filament from a denser droplet grows at a higher speed. From the current simulation, the earlier thermal behavior of the droplet agrees with Ciccarelli and Frost's model [2]; Kim's model [7] is not observed. It is believed that the model of Ciccarelli and Frost [2] is a more reasonable one to describe the thermal behavior of a molten droplet.

At the later stage of the propagation, the hydrodynamic effect will act as a dominant role. For the earlier hydrodynamic behavior, the study of film thickness indicates that the presence of vapor film will suppress the droplet deformation generally. The effect of molten droplet density is also studied and it shows that the denser droplet experiences larger deformation. Particularly, the simulation results of external triggering pressure pulse reveal that vapor film will have little effect on the droplet deformation when the amplitude of the pressure pulse is very high.

Considering the effect of the vapor film on droplet behavior during the process of steam explosion, the presence of vapor film has effect on droplet behavior at the stage of triggering and the early stage of the propagation, because the thermal effect is dominant at these stages. When the pressure pulse is coupled to high amplitude, which is the situation at the later stage of the propagation, pure hydrodynamic effect is dominant. Due to current study, the effect of vapor film can be neglected.

\section{Nomenclature}

$\begin{array}{ll}C: & \text { Fractional volume } \\ C_{P}: & \text { Specific heat } \\ d: & \text { Diameter/deformation displacement } \\ h_{l v}: & \text { Latent heat } \\ L: & \text { Wave amplitude } \\ m: & \text { Mass } \\ \vec{n}: & \text { Unit normal vector } \\ n: & \text { Fastest growth for Rayleigh-Taylor instability } \\ p: & \text { Pressure } \\ R: & \text { Radius } \\ T: & \text { Temperature } \\ u, v, V: \text { Velocity } \\ \text { We: } & \text { Weber number. }\end{array}$

\section{Greek Symbols}

$\delta:$ Dirac distribution/film thickness

$\kappa$ : Curvature

$\rho$ : Density

$\varepsilon$ : Radiation emissivity

$\sigma$ : Surface tension

$\sigma_{b}$ : Stefan-Boltzmann constant

$\lambda$ : Wave length/conductivity

$\mu$ : Dynamic viscosity.

\section{Subscripts \\ 0: Initial \\ d: $\quad$ Molten droplet \\ film: Vapor film \\ c: Coolant \\ $i$ : $\quad$ Phase index \\ $v$ : Vapor.}

\section{Acronyms}

CSF: Continuum Surface Force

FCI: Fuel and Coolant Interaction

NPP: Nuclear Power Plant

PLIC: Piecewise Linear Interface Construction.

\section{Conflict of Interests}

The authors declare that there is no conflict of interests regarding the publication of this paper.

\section{Acknowledgment}

Authors are grateful to National Science and Technology Major Project (2011ZX06004-024) of China for providing the financial support for this study.

\section{References}

[1] Y. Abe, E. Matsuo, T. Arai et al., "Fragmentation behavior during molten material and coolant interactions," Nuclear Engineering and Design, vol. 236, no. 14-16, pp. 1668-1681, 2006.

[2] G. Ciccarelli and D. L. Frost, "Fragmentation mechanisms based on single drop steam explosion experiments using flash X-ray radiography," Nuclear Engineering and Design, vol. 146, no. 1-3, pp. 109-132, 1994.

[3] M. Pilch and C. A. Erdman, "Use of breakup time data and velocity history data to predict the maximum size of stable fragments for acceleration-induced breakup of a liquid drop," International Journal of Multiphase Flow, vol. 13, no. 6, pp. 741757, 1987.

[4] L. S. Nelson and P. M. Duda, "Steam explosion experiments with single drops of iron oxide melted with a $\mathrm{CO}_{2}$ laser," NUREG/ CR-2295, SAND 81-1346 R3, Sandia Natl. Lab., Albuquerque, NM, USA, 1981.

[5] H. S. Park, R. C. Hansson, and B. R. Sehgal, "Fine fragmentation of molten droplet in highly subcooled water due to vapor explosion observed by X-ray radiography," Experimental Thermal and Fluid Science, vol. 29, no. 3, pp. 351-361, 2005. 
[6] T. Takashima, "Observations of initiation stage of spontaneous vapor explosions for droplet scale," Heat Transfer-Asian Research, vol. 37, no. 1, pp. 41-55, 2008.

[7] B. Kim and M. L. Corradini, "Modeling of small-scale single drop fuel coolant interactions," Nuclear Science and Engineering, vol. 98, no. 1, pp. 16-28, 1988.

[8] S. Koshizuka, H. Ikeda, and Y. Oka, "Numerical analysis of fragmentation mechanisms in vapor explosions," Nuclear Engineering and Design, vol. 189, no. 1, pp. 423-433, 1999.

[9] Y. Zhou, Z. Zhang, M. Lin, M. H. Yuan, and X. Yan, "Numerical simulation of fragmentation of melt drop triggered by external pressure pulse in vapor explosions," Annals of Nuclear Energy, vol. 57, pp. 92-99, 2013.

[10] S. J. Board, R. W. Hall, and R. S. Hall, "Detonation of fuel coolant explosions," Nature, vol. 254, no. 5498, pp. 319-321, 1975.

[11] D. D. Joseph, J. Belanger, and G. S. Beavers, "Breakup of a liquid drop suddenly exposed to a high-speed airstream," International Journal of Multiphase Flow, vol. 25, no. 6-7, pp. 1263-1303, 1999.

[12] R. C. Hansson, H. S. Park, and T.-N. Dinh, "Dynamics and preconditioning in a single-droplet vapor explosion," Nuclear Technology, vol. 167, no. 1, pp. 223-234, 2009.

[13] S. Thakre, W. Ma, and L. Li, "A numerical analysis on hydrodynamic deformation of molten droplets in a water pool," Annals of Nuclear Energy, vol. 53, pp. 228-237, 2013.

[14] M. J. Zhong, Y. Zhou, M. Lin, M. H. Yuan, and Y. H. Yang, "Numerical simulation of molten droplet deformation and disintegration under sudden accelerations," Annals of Nuclear Energy, vol. 65, pp. 199-206, 2014.

[15] G. Berthoud, "Vapor explosions," Annual Review of Fluid Mechanics, vol. 32, pp. 573-611, 2000.

[16] J. U. Brackbill, D. B. Kothe, and C. Zemach, "A continuum method for modeling surface tension," Journal of Computational Physics, vol. 100, no. 2, pp. 335-354, 1992.

[17] D. B. Kothe and R. C. Mjolsness, "RIPPLE: a new model for incompressible flows with free surfaces," AIAA journal, vol. 30, no. 11, pp. 2694-2700, 1992.

[18] J. R. Richards, A. N. Beris, and A. M. Lenhoff, "Drop formation in liquid-liquid systems before and after jetting," Physics of Fluids, vol. 7, no. 11, pp. 2617-2630, 1995.

[19] M. H. Yuan, Y. H. Yang, T. S. Li, and Z. H. Hu, "Numerical simulation of film boiling on a sphere with a volume of fluid interface tracking method," International Journal of Heat and Mass Transfer, vol. 51, no. 7-8, pp. 1646-1657, 2008.

[20] N. I. Kolev, "Film boiling on vertical plates and spheres," Experimental Thermal and Fluid Science, vol. 18, no. 2, pp. 97-115, 1998.

[21] N. Ashgriz, "FLAIR: Flux line-segment model for advection and interface reconstruction," Journal of Computational Physics, vol. 93, no. 2, pp. 449-468, 1991.

[22] D. L. Youngs, “Time-dependent multi-material flow with large fluid distortion," Numerical Methods for Fluid Dynamics, vol. 24, pp. 273-285, 1982.

[23] T. R. Fodemski, "Forced convection film boiling in the stagnation region of a molten drop and its application to vapour explosions," International Journal of Heat and Mass Transfer, vol. 35, no. 8, pp. 2005-2016, 1992.

[24] R. C. Hansson, H. S. Park, and T.-N. Dinh, "Simultaneous high speed digital cinematographic and $\mathrm{X}$-ray radiographic imaging of a intense multi-fluid interaction with rapid phase changes," Experimental Thermal and Fluid Science, vol. 33, no. 4, pp. 754$763,2009$.
[25] D. Buxton, L. S. Nelson, and W. B. Benedick, Steam Explosion Triggering and Efficiency Studies, Sandia Labs, Albuquerque, NM, USA, 1979.

[26] V. M. Boiko and S. V. Poplavski, "On the dynamics of drop acceleration at the early stage of velocity relaxation in a shock wave," Combustion, Explosion and Shock Waves, vol. 45, no. 2, pp. 198204, 2009.

[27] M. Leskovar and M. Uršič, "Estimation of ex-vessel steam explosion pressure loads," Nuclear Engineering and Design, vol. 239, no. 11, pp. 2444-2458, 2009. 


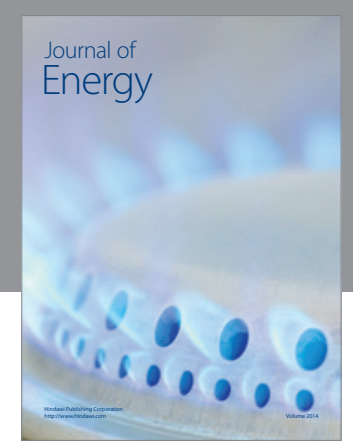

Journal of

Industrial Engineering
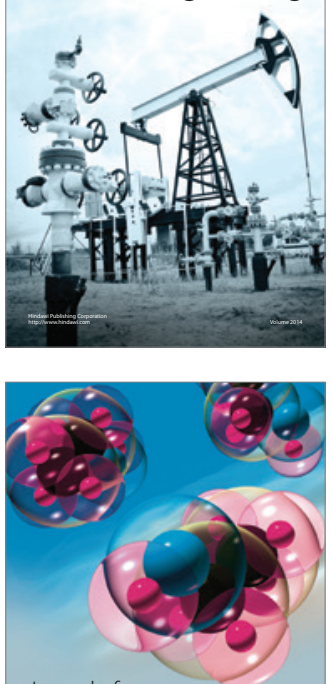

Fuels
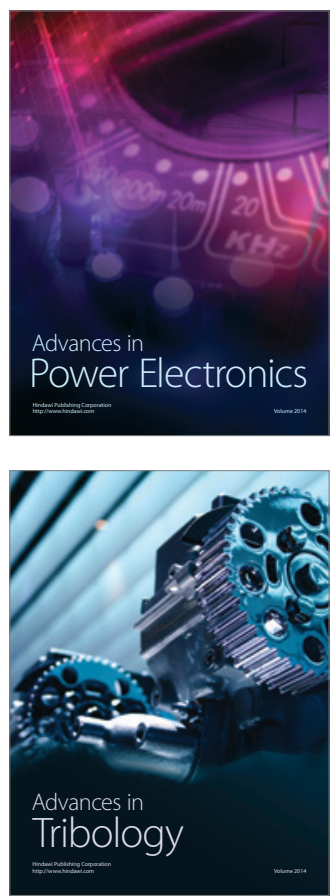

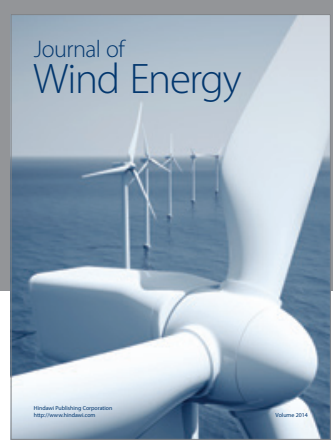

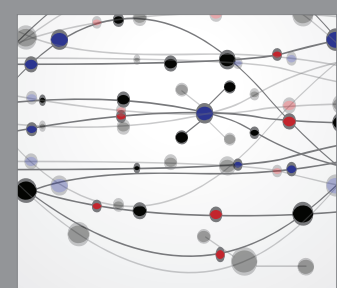

The Scientific World Journal

Submit your manuscripts at http://www.hindawi.com

Journal of

Structures
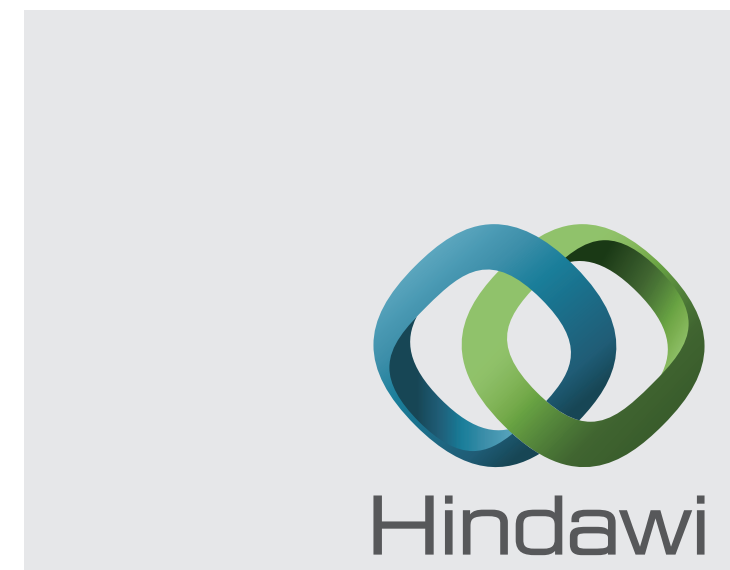

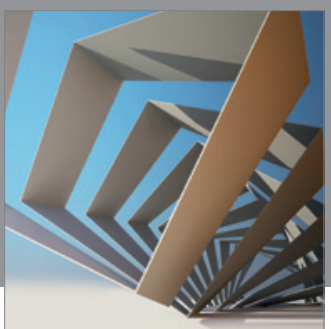

Rotating

Machinery
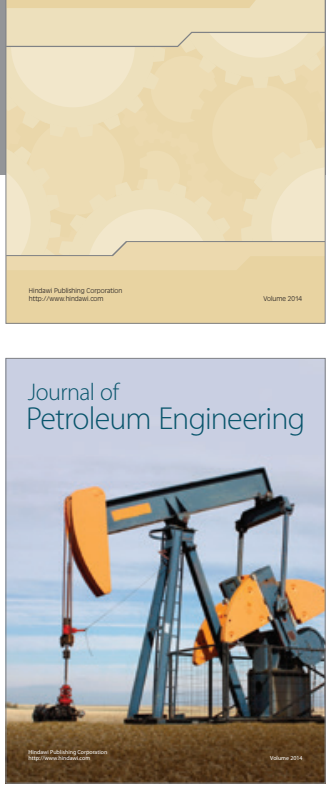

Journal of

Solar Energy
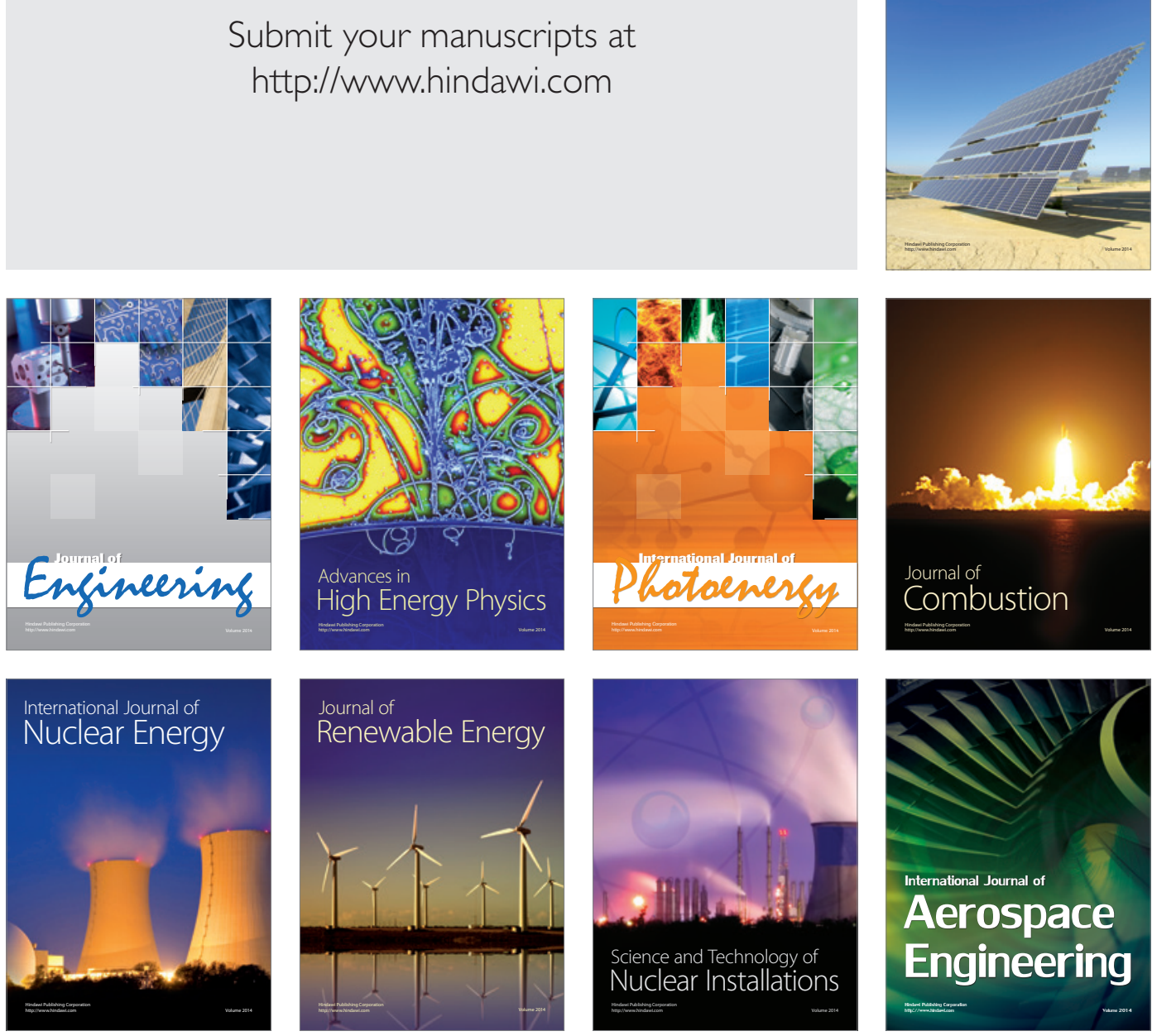